\title{
ANÁLISE DE PADRÃO DE PRESCRIÇÃO ODONTOLÓGICA EM UNIDADES DE SAÚDE PÚBLICA DE UM MUNICÍPIO DE MATO GROSSO DO SUL, BRASIL.
}

\author{
ANALYSIS OF PRESCRIBING PATTERN IN DENTAL PUBLIC HEALTH IN A UNIT OF \\ A CITY OF MATO GROSSO DO SUL, BRAZIL.
}

\author{
Adriana Mary Mestriner Felipe de Meloํㅜㄹ José Eduardo Barbosa Mestriner2; Marcos \\ Ávalos Berndt ${ }^{3}$; Maria Beatriz Cardoso Ferreira ${ }^{4}$
}

\begin{abstract}
1 - Doutora em Ciências da Saúde (UnB), Mestre em Microbiologia (UEL), docente da Unigran
2- Odontólogo, Especialista em Saúde Coletiva pela Escola Davi Nasser

3 - Farmacêutico, Mestre em Ciências Farmacêuticas (UFMS)

4 - Pós Doutora, Pesquisadora associada Docente, CAPES
\end{abstract}

\section{RESUMO:}

A prescrição é uma das ferramentas na promoção do uso seguro e racional de medicamentos. O objetivo desta pesquisa foi investigar a prescrição de medicamentos por Cirurgiões-dentistas a pacientes atendidos em nove unidades de atendimento odontológico da rede de assistência pública de Naviraí (MS). Foi realizado estudo retrospectivo no período de janeiro a junho de 2009, tendo como base 923 receituários obtidos por meio de cópias carbonadas. Avaliaram-se os indicadores de prescrição para uso racional de medicamentos, preconizados pela Organização Mundial de Saúde (OMS). A utilização de dois ou mais medicamentos numa mesma prescrição foi considerado como polifarmácia. Ao todo, foram prescritos 1.322 medicamentos. A maioria das prescrições continha informações de posologia completa, abrangendo forma farmacêutica, intervalo e tempo do tratamento. Dentre os indicadores pesquisados, o número médio de medicamentos por prescrição foi de 1,4 , sendo que $96 \%$ foram prescritos sob denominação genérica e $63 \%$ estavam contemplados na Relação Nacional de Medicamentos Essenciais. A Frequência de polifarmácia foi considerada baixa. Observou-se, ainda, que $45 \%$ das prescrições continham pelo menos um medicamento antimicrobiano, percentual considerado elevado. Penicilinas foram a classe de antimicrobiano mais prescrita. No entanto, em apenas $2,4 \%$ das prescrições, havia a indicação de pelo menos um medicamento injetável. A maioria dos indicadores de prescrição estabelecidos pela OMS foi atendida, sugerindo um Uso Racional de Medicamentos pelos cirurgiões-dentistas no atendimento a população.

Palavras-chave: Prescrição, Medicamentos, dentistas.

\begin{abstract}
:
Prescription is one of the tools in promoting the safe and rational use of medicines. The objective of this research was to investigate the prescription of medications by dental surgeons to patients seen in nine dental care units in the public assistance network of Naviraí (MS). A retrospective study was carried out from January to June 2009, based on 923 prescriptions obtained through carbonated copies. Prescription indicators for rational use of medicines, recommended by the World Health Organization (WHO), were evaluated. The use of two or more drugs in the same prescription was considered to be polypharmacy. In total, 1,322 drugs were prescribed. Most prescriptions contained complete dosage information, covering dosage form, interval and time of treatment. Among the indicators surveyed, the average number of drugs per prescription was $1.4,96 \%$ of which were
\end{abstract}


prescribed under a generic name and $63 \%$ were included in the National List of Essential Medicines. Polypharmacy frequency was considered low. It was also observed that $45 \%$ of the prescriptions contained at least one antimicrobial medication, a percentage considered high. Penicillins were the most prescribed antimicrobial class. However, in only $2.4 \%$ of prescriptions, there was an indication of at least one injectable medication. Most of the prescription indicators established by the WHO were met, suggesting a Rational Use of Medicines by dentists in serving the population.

Keywords: Prescription, Dentist, Drugs.

\section{INTRODUÇÃO}

O uso racional de medicamentos é uma prática difundida a fim de aperfeiçoar a escolha do medicamento apropriado para a condição clínica do paciente adaptando-se a dose, período e acesso ao tratamento (OMS, 1986). Nesse processo, apesar de diferentes atores estarem envolvidos (QUELUZ, 2008) o prescritor é peça de destaque, por ter a atribuição de transcrever ao paciente essas informações por meio da prescrição (MARIN; MACHADO, 2008).

A prescrição trata-se do documento formal e escrito que estabelece o que deve ser dispensado ao paciente e como ele será utilizado (OMS, 1998), respeitando a individualidade associado ao binômio custo-benefício (BRASIL, 2016). Dentre os profissionais habilitados à prescrição medicamentosa, o cirurgião-dentista possui esta competência quando se fizer necessário (BRASIL, 1966; CONSELHO REGIONAL DE ODONTOLOGIA DE SÃO PAULO, 2019). Dado a importância do tema, a prescrição de medicamentos por esses profissionais tem sido foco de inúmeras pesquisas e discussões em todo o mundo. Em Belo Horizonte (MG), um estudo com 163 cirurgiões-dentistas revelou que a maior parte dos medicamentos era prescrita pelo nome comercial e não, pela denominação Comum Brasileira (DCB) (CASTILHO; PAIXÃO; PERINI, 1999).

Pensando que falhas durante a prescrição podem ocasionar danos à saúde do paciente (SANTOS; ROCHA; SAMPAIO, 2019) existe uma preocupação crescente no que diz respeito à profilaxia antimicrobiana, pois pode interferir na resistência aos agentes antimicrobianos. No Canadá, Epstein \& Chong (2000) encontraram que 19,9\% dos 2.542 profissionais que responderam ao inquérito confirmaram que a classe das penicilinas foi empregada como antibióticos de primeira escolha após o tratamento odontológico. Lauber e colaboradores (2007) encontraram adesão dos profissionais as condutas preconizadas para os pacientes com endocardite bacteriana utilizando amoxicilina como antibiótico de primeira escolha (na dose de $2 \mathrm{~g}$, uma hora antes do tratamento odontológico). Já Palmer e colaboradores (2001) evidenciaram a precariedade de conhecimento entre os 
profissionais da Inglaterra e Irlanda, destacando que medidas de qualificação nessa área são necessárias. Contudo, a pesquisa de Castilho e colaboradores (1999) mostrou que os treinamentos na área de farmacologia se não realizados da forma correta acabam não refletindo em mudança de conduta na prática de prescrição odontológica. Assim, uma das estratégias válidas é de que essas atualizações utilizem estratégias inovadoras e não metodologias convencionais.

Novas publicações procuram disponibilizar informações que facilitem a prescrição correta de medicamentos orais para os cirurgiões-dentistas aperfeiçoa o atendimento, aumentando o sucesso terapêutico, minimizando riscos e adequando o uso de medicamentos à necessidade de cada paciente. Além disso, outros documentos têm sido produzidos para facilitar o emprego de terapias adequadas (DAR-ODEH et al., 2010) do ponto de vista do uso racional e correto de medicamentos (TULLY; CANTRILL, 2006), especialmente tratando-se de grupos vulneráveis, como gestantes e lactantes (AMADEI et al., 2011).

Considerando a prescrição como um instrumento importante no uso racional de medicamentos, o objetivo desta pesquisa foi identificar a prática de prescrição pelos cirurgiões-dentistas que atuam nas Unidades da Rede Municipal de um município do interior de Mato Grosso do Sul.

\section{MATERIAL E MÉTODOS}

Foi realizado estudo descritivo-transversal e retrospectivo de indicadores de uso racional de medicamentos em cópias das prescrições de cirurgiões-dentistas da rede pública de Naviraí (MS). A pesquisa foi desenvolvida entre o período de janeiro a junho de 2009.

A pesquisa foi realizada no município de Naviraí, localizado na região sul do estado de Mato Grosso do Sul, na microrregião de Iguatemi tendo a sua posição astronômica em $23^{\circ} 03^{\prime} 45^{\prime \prime}$ de Latitude Sul e 54 $11^{\prime}$ '26" de Longitude Oeste, com uma área de 3.194 km² e população estimada em 45.627 habitantes (IBGE, 2010). O atendimento odontológico público disponibilizado pela prefeitura municipal de Naviraí/MS é realizado em um Centro Odontológico e oito unidades da Estratégia de Saúde da Família (ESF), contando cada uma delas com uma equipe de saúde bucal e um cirurgião-dentista. As oito unidades de ESF fazem cobertura em áreas circunscritas, com atendimento aproximado de setenta por cento da população urbana. O Centro Odontológico conta com cinco cirurgiões-dentistas para 
prestar atendimento às áreas descobertas pelos ESF e, também, oferece serviços especializados a todo o município.

O projeto foi submetido e aprovado pelo Comitê de Ética e Pesquisa da Universidade Federal do Mato Grosso do Sul (Protocolo 1521/2009), seguindo as normativas éticas previstas na Resolução do Conselho Nacional de Saúde para pesquisa envolvendo Seres Humanos. O estudo não interferiu com o tratamento proposto ou com as prescrições medicamentosas e não medicamentosas recebidas pelos pacientes, visto que o objeto da pesquisa foram cópias carbonadas.

O estudo avaliou 923 cópias carbonadas das prescrições emitidas pelos cirurgiõesdentistas nas equipes de ESF e no Centro Odontológico. A prática de carbonar o receituário já fazia parte da rotina das unidades previamente a proposição do estudo. Foram inseridas na pesquisa todas as prescrições emitidas pelos cirurgiões-dentistas das unidades acima caracterizadas da rede municipal de saúde de Naviraí (MS), enquanto aquelas provenientes de eventuais atendimentos na zona rural do município foram excluídas.

O instrumento de coleta de dados foi construído utilizando-se como referência os indicadores de Prescrição de Uso Racional de Medicamentos adotados pela OMS baseados no manual "Guia para Boa Prescrição Médica", conforme descrito a seguir:

1) Número médio de medicamentos por prescrição (determina o grau de poli medicação a que a população está sujeita) = total de medicamentos prescritos/ total de prescrições envolvidas na pesquisa;

2) Porcentagem de medicamentos prescritos pelo nome genérico (determina a tendência do prescritor para optar por nomes genéricos na prescrição) $=$ total de medicamentos genéricos prescritos/ total de medicamentos prescritos, multiplicando-se por 100.

3) Porcentagem de receitas em que se prescrevem antibióticos (determina o nível de emprego de antibióticos nos esquemas terapêuticos, o que, normalmente, tem maior custo, utilização complexa e são objeto de abuso) = receitas em que foi prescrito pelo menos um antibiótico/total de receitas, multiplicando- se por 100.

4) Porcentagem de receitas em que se prescrevem injetáveis (determina o grau de utilização destas modalidades de tratamento que, normalmente, são de maior custo, utilização complexa e passíveis de abuso) = receitas em que foi prescrito ao menos um injetável/total de receitas, multiplicando-se por 100.

5) Porcentagem de medicamentos prescritos que pertencem à Relação Nacional de Medicamentos Essenciais (determina a adequação do serviço à Política Nacional de Medicamentos) $=$ total de medicamentos prescritos que figuram na RENAME (Relação 
Nacional de Medicamentos Essenciais) /total de medicamentos prescritos, multiplicandose por 100 .

6) Porcentagem de medicamentos prescritos que pertencem à Lista de Medicamentos Essenciais da OMS (determina a adequação do serviço as recomendações da Oms) = total de medicamentos prescritos que figuram na Lista de Medicamentos Essenciais da Oms/ total de medicamentos prescritos, multiplicando-se por 100.

Estes indicadores refletem o funcionamento do serviço de saúde, principalmente no que se refere às práticas gerais de prescrição, além da presença ou não dos dados do prescritor, nome do paciente. Outros itens relacionados ao medicamento também foram avaliados, tais como: denominação do medicamento, via e intervalo de administração, forma farmacêutica, concentração e tempo de tratamento.

Os medicamentos prescritos em cada receita foram listados por seus nomes genéricos e classificados obedecendo à classificação Anatômica, química e terapêutica (Anatomical Therapeutic Chemical Classification - ATC), da Organização Mundial de Saúde, para os estudos de utilização de medicamentos. Tal classificação permitiu a definição das classes de medicamentos mais prescritas nos serviços odontológicos do município.

Os dados foram reunidos, codificados e analisados em banco de dados por meio do programa SPSS versão 16.0 for Windows (SPSS Inc., Chicago, Estados Unidos). Os resultados foram expressos como frequência absoluta e frequência relativa.

\section{RESULTADOS E DISCUSSÃO}

Foram analisadas 923 prescrições provenientes de 9 locais onde são prestados serviços odontológicos que fazem parte do Sistema Único de Saúde (SUS) em Naviraí (MS). Destas 923 prescrições, 551 (60 \%) eram de pacientes do gênero feminino e 372 (40\%) do gênero masculino.

O nome do paciente estava presente em todas as prescrições analisadas, contudo, o endereço estava ausente, contrariando o Art. 35, inciso III do Decreto Presidencial $n^{\circ} 793$, de 5 de Abril de 1993 que obriga a descrição do nome e endereço do paciente nas prescrições. O nome do prescritor estava presente em 306 (33 \%) prescrições, enquanto o número de inscrição no Conselho da Classe (Conselho Regional de Odontologia - CRO) foi transcrito em um número ainda menor, 103 (11\%). Já a assinatura do prescritor e a data da prescrição estiveram presentes em mais de $97 \%$ das prescrições, seguido do endereço, 
462 (50\%). Cabe mencionar que o objeto de estudo foram as cópias carbonadas das prescrições recebidas pelo paciente, o que pode não significar que os dados do prescritor não estavam presentes, visto que o uso de carimbos para esse tipo de informação é prática frequente que não permite a transcrição na cópia carbonada.

Ao todo, dos 1322 medicamentos prescritos, aproximadamente 99\% apresentavam a forma farmacêutica e $87 \%$ continham a posologia completa. A presença da dose e intervalo foi mencionada em $97 \%$ das prescrições, enquanto a duração do tratamento em $88 \%$ (tabela 1), itens que atendem a boa prática de prescrição medicamentosa (OMS, 1998).

Um dado positivo foi que em 96\% (1274) dos medicamentos prescritos o nome genérico foi utilizado. A forma farmacêutica foi mencionada em praticamente todas as prescrições (99\%), sendo que as mais frequentes foram os comprimidos (47\%), cápsulas (25\%) e gotas (17\%). Apesar de não estar expressa, subentendeu-se através da forma farmacêutica indicada que em 1232 (93,2\%) dos medicamentos a indicação foi para via oral e em menor proporção para uso tópico (65 ou 4,9\%) e parenteral (25 ou 1,9\%). Um percentual reduzido de medicamentos injetáveis (2\%) já era esperado por não se tratar de ambiente hospitalar.

Mais da metade dos medicamentos prescritos $(840 ; 63 \%)$ atendiam a Relação Nacional de Medicamentos Essenciais (RENAME). Enquanto o número médio de medicamentos foi de 1,43 por prescrição. Houve predomínio de um ou dois medicamentos por prescrição, em 95\% do total de prescrições (tabela 1).

Tabela 1- Medicamentos prescritos de acordo com a Classificação Anatômica, Química e Terapêutica (ATC) em prescrições odontológicas da rede Municipal de Saúde de Naviraí/MS, janeiro a junho de 2009.

\begin{tabular}{lcccc}
\hline \multicolumn{1}{c}{ Agentes } & Classe & Classificação & \multicolumn{2}{c}{ Frequência } \\
\cline { 4 - 6 } farmacológicos & Farmacológica & ATC & $\mathbf{n}$ & $\mathbf{( \% )}$ \\
\hline Amoxicilina & Antibiótico de uso sistêmico & J01CA04 & 365 & 27,6 \\
Diclofenaco & Anti-inflamatório não esteroidal & M01AB05 & 315 & 23,4 \\
Paracetamol & Analgésico & N02BE01 & 243 & 18,4 \\
Dipirona & Analgésico & N02BB02 & 173 & 13,0 \\
lbuprofeno & Anti-inflamatório não esteroidal & M01AE01 & 51 & 3,8 \\
\hline
\end{tabular}

Dentre os medicamentos mais prescritos, o diclofenaco (23\%) foi o segundo mais prescrito (tabela 4). 
Já a presença de pelo menos um medicamento antimicrobiano foi encontrado em 417 prescrições (45 \%), com predomínio das penicilinas (amoxicilina), tanto como único fármaco quanto em associação com outras drogas (tabelas 2 e 3).

O predomínio de prescrições destinadas a pacientes do sexo feminino foi maior também no estudo realizado na Índia por Sarkar e Baral (2004). Esse dado deve-se, talvez, pela utilização dos serviços de saúde ser maior das mulheres do que do sexo oposto.

Quanto analisado os itens constantes nas recomendações de boas práticas de prescrição medicamentosa (OMS, 1998), os resultados foram satisfatórios.

Tabela 2 - Associação medicamentosa encontrada nas prescrições odontológicas da rede Municipal de Saúde de Naviraí/MS, janeiro a junho de 2009.

\begin{tabular}{lll}
\hline \multirow{2}{*}{ Agentes farmacológicos } & \multicolumn{2}{c}{ Frequência } \\
\cline { 2 - 3 } & Absoluta (n) & relativa (\%) \\
\hline amoxicilina + paracetamol & 72 & 7,80 \\
amoxicilina + diclofenaco & 56 & 6,07 \\
amoxicilina + dipirona & 31 & 3,36 \\
amoxicilina + metronidazol & 22 & 2,38 \\
amoxicilina + ibuprofeno & 12 & 1,30 \\
amoxicilina + diclofenaco + dipirona & 8 & 0,87 \\
amoxicilina + diclofenaco + paracetamol & 7 & 0,76 \\
diclofenaco + paracetamol & 54 & 5,85 \\
diclofenaco + dipirona & 28 & 3,03 \\
azitromicina + dipirona & 8 & 0,87 \\
\hline
\end{tabular}

Tabela 3 - Indicadores de Uso Racional de Medicamentos presentes nas prescrições odontológicas de da rede Municipal de Saúde de Naviraí/MS, janeiro a junho de 2009.

\begin{tabular}{|c|c|c|c|}
\hline \multirow[b]{2}{*}{ Indicadores de prescrição } & \multicolumn{2}{|c|}{ Valores } & \multirow{2}{*}{ Média (\%) } \\
\hline & $\mathrm{n}$ & $\mathbf{N}$ & \\
\hline Número de medicamentos por prescrição & 1322 & 923 & $1,43^{* *}$ \\
\hline $\begin{array}{l}\text { Porcentagem de prescrições contendo pelo menos um medicamento } \\
\text { antimicrobiano }\end{array}$ & 417 & 923 & 45,2 \\
\hline $\begin{array}{l}\text { Porcentagem de prescrições contendo pelo menos um medicamento } \\
\text { injetável }\end{array}$ & 25 & 923 & 2,7 \\
\hline Porcentagem de medicamentos prescritos pelo nome genérico & 1274 & 1322 & 96,4 \\
\hline $\begin{array}{l}\text { Porcentagem de medicamentos prescritos (nome e apresentação) } \\
\text { constantes da lista nacional de medicamentos (RENAME) }\end{array}$ & 840 & 1322 & 63,5 \\
\hline $\begin{array}{l}\text { Porcentagem de medicamentos prescritos (nome e apresentação) } \\
\text { constantes da lista modelo de medicamentos essenciais da OMS }\end{array}$ & 642 & 1322 & 48,5 \\
\hline
\end{tabular}

$\mathrm{N}$ : amostra total; n: número encontrado relacionados com o indicador referido; ${ }^{* *}$ não está em porcentagem $\mathrm{e}$ sim em média aritmética. Assim se lê: 1,43 medicamentos por prescrição. 
Apesar dos resultados terem sido satisfatórios quanto à adequação a RENAME, a Relação Municipal de Medicamentos Essenciais (REMUME) não pode ser utilizada no estudo, visto que o município em estudo ainda não possuía a lista de forma oficial, com elaboração por uma comissão de farmacoterapêutica. No momento do estudo, a escolha e compra dos medicamentos era realizada por meio de previsões analíticas das indicações dos prescritores (médicos e odontólogos), bem como a demanda epidemiológica e sazonal. Diante do observado em Naviraí, é necessário que seja elaborada e implementada a Relação Municipal de Medicamentos Essenciais (REMUME) por uma Comissão de Farmacoterapêutica, como forma de otimizar o tratamento, custos, disponibilidade e acesso da população à medicação.

Considerando a recomendação da OMS de 1,33 a 2,0 medicamentos devem ser prescritos por prescrição/ paciente para ser indicativo de não poli medicação, o estudo constatou resultados bastante pertinentes, visto que o número médio constatado foi de 1,43 .

Apesar de o diclofenaco ter sido o segundo medicamento mais prescrito (tabela 4), existe outras opções para a classe dos anti-inflamatórios não esteroidais previstos para uso na prática odontológica, como o ácido acetilsalicílico e ibuprofeno (BRASIL, 2007).

O uso da amoxicilina (500 mg, de 8 em 8 horas ou 2 g, de 12 em 12 horas, associado ao ácido clavulânico, $125 \mathrm{mg}$ ) é uma das opções terapêuticas antimicrobianas empregadas na pratica odontológica (ISLA et al., 2005). O número expressivo do uso de pelo menos um antimicrobiano nas prescrições pode ser correlacionado ao fato de que o cirurgião-dentista realiza procedimentos profiláticos com antimicrobianos além da terapêutica de doenças infectocontagiosas propriamente ditas. É importante destacar que a seleção é realizada utilizando-se dados epidemiológicos, pois o profissional raramente realiza a cultura de micro-organismos nos exsudados provenientes das lesões orais.

A indicação do medicamento prescrito pelo nome genérico atendeu às recomendações da OMS (OMS, 1998) e as legislações brasileiras (BRASIL, 1993, 1999) que determinam que no âmbito do Sistema Único de Saúde (SUS) todas as prescrições devem ser feitas pelos nomes genéricos nos receituários médicos e odontológicos. Resultados diferentes daqueles encontrados em estudo semelhante realizado em Belo Horizonte (MG) (CASTILHO; PAIXÃO; PERINI, 1999) em que os medicamentos foram mais prescritos pelo nome comercial, com tendência a prescrever mais frequentemente antiinflamatórios em relação aos analgésicos. 


\section{CONCLUSÕES}

Considerando a importância da prescrição odontológica no uso racional de medicamentos, o presente estudo visou contribuir para que administradores da saúde identifiquem a realidade local de modo que possam programar políticas de saúde na área. A amoxicilina (J01CA04), o diclofenaco (M01AB05) e o paracetamol (N02BE01) foram os medicamentos mais prescritos de acordo com a Classificação Anatômica, Química e Terapêutica (ATC).

De uma forma geral as prescrições odontológicas da rede municipal do Município de Naviraí atendem a maioria dos indicadores de prescrição da OMS, bem como as recomendações presentes no Manual de Boas prescrições.

\section{REFERÊNCIAS}

BRASIL. Lei 5.081 de 24/08/1966. Regula o Exercício da Odontologia. Diário Oficial da República Federativa do Brasil, Brasília (DF); 1966.

BRASIL. Decreto 793, de 5 de abril de 1993. Altera os Decretos $n^{\circ} 74170$, de 10 de junho de 1974 e 79.094 de 5 de janeiro de 1977, que regulamentam, respectivamente, as Leis $\mathrm{n}^{\circ}$ 5991, de 17 de janeiro de 1973, e 6360 de 23 de setembro de 1976, e dá outras providências. Diário Oficial da República Federativa do Brasil, Brasília (DF); 1993; abr 6; Seção 1: 4397.

BRASIL. Lei 9787 de 10 de fevereiro de 1999. Altera a lei $n^{\circ} 6360$ de 23 de setembro de 1976, que dispõe sobre a vigilância sanitária, estabelece o medicamento genérico, dispõe sobre a utilização de nomes genéricos em produtos farmacêuticos e dá outras providências. Diário Oficial da República Federativa do Brasil, Brasília (DF); fev 11; Seção 1:1. 1999.

BRASIL. Ministério da Saúde. Secretaria de Políticas de Saúde. Departamento de Atenção Básica. Gerência Técnica de Assistência Farmacêutica. Brasília: Ministério da Saúde, 2007.

BRASIL. Relação Nacional de Medicamentos Essenciais (Rename). 5. ed. Brasília: Ministério da Saúde, 2007. 
BRASIL. Ministério da Saúde. Secretaria de Ciência, Tecnologia e Insumos Estratégicos. Departamento de Ciência e Tecnologia. Síntese de evidências para políticas de saúde: adesão ao tratamento medicamentoso por pacientes portadores de doenças crônicas / Ministério da Saúde, Secretaria de Ciência, Tecnologia e Insumos Estratégicos, Departamento de Ciência e Tecnologia. Brasília: Ministério da Saúde, 2016. 52 p.

CASTILHO Lia S, Helena H. Paixão e Edson Perini Prescrição de medicamentos de uso sistêmico por cirurgiões dentistas, clínicos gerais Rev. Saúde Pública, v. 33, n. 3, p. 28794, 1999.

CODATO, Lucimar Aparecida Britto et al. Atenção odontológica à gestante: papel dos profissionais de saúde. Ciênc. saúde coletiva, Rio de Janeiro, v. 16, n. 4, p. 22972301, 2011.

CONSELHO REGIONAL DE ODONTOLOGIA DE SÃO PAULO. Prescrição e dispensação de medicamentos na odontologia: Guia Prático. Vol. 4. Disponível em <http://www.crosp.org.br/ uploads/arquivo/c2035e1fd371097aa1de449aceca1291.pdf> acesso em 21 de dezembro de 2019.

DAR-ODEH, Najla Saeed et al. Antibiotic prescribing practices by dentists: a review. Therapeutics and clinical risk management, v. 6, p. 301-6, 2010.

EPSTEIN, J.B.; CHONG, S.; LE, N.D. A survey of antibiotic use in dentistry. JADA, v. 131, p. $1600-9,2000$.

GOMES, M.J.V.M.; REIS, A.M.M., MAGALHÃES, J.B.S. Ciências Farmacêuticas: Uma Abordagem em Farmácia Hospitalar. São Paulo: Editora Atheneu, 2003.

INSTITUTO BRASILEIRO DE GEOGRAFIA E ESTATÍSTICA (IBGE). Disponível em <http://www.ibge.gov.br> acesso em 11 de fevereiro de 2019.

ISLA A et al. Farmacocinética/ farmacodinámica de la formulacion de amoxicilina/acido clavulanico 1000/ 62,5 mg en odontoestomatologia. Enferm. Infecc. Microbiol, v. 23, p. 387, 2005. 
LAUBER, Carmen et al. Antibiotic prophylaxis practices in dentistry: A survey of dentists and physicians. Journal Canadian Dental Association, v. 73, n.3, p. 245-263, 2007.

MARIN, Nelly. (org.) Assistência farmacêutica para gerentes municipais. / Organizado por Nelly Marin et al. Rio de Janeiro: OPAS/OMS, 2003. 373p. Disponível em: <htpp://www.opas.org.br/medicamentos/>. Acesso em 15/mar/2020.

ORGANIZAÇÃO MUNDIAL DA SAÚDE (OMS). Guia para Boa Prescrição Médica. Porto Alegre: Artmed, 1998.

ORGANIZAÇÃO MUNDIAL DA SAÚDE (OMS). Uso Racional de los Medicamentos. Informe de la Conferencia de Expertos. Nairobi, 25-29 de noviembre de 1985. Genebra: Organización Mundial de la Salud, 1986.

PALMER, N.O.A., DAILEY, Y.M. General dental practitioners' experiences of a collaborative clinical audit on antibiotic prescribing: a qualitative study. British Dental Journal, v. 193, n.1, p.46-9, 2002.

QUELUZ, T.H.A.T., LEITE, S.N. Uso Racional de medicamentos: conceitos e alguns elementos para discussão. In: Cordeiro BC, Leite SN (Org.). 0 farmacêutico na atenção à Saúde. Itajaí: Univalli, 2008. p.1-25.

SANTOS, Patricia Reis Alves dos; ROCHA, Fernanda Ludmilla Rossi; SAMPAIO, Camila Santana Justo Cintra. Ações para segurança na prescrição, uso e administração de medicamentos em unidades de pronto atendimento. Rev. Gaúcha Enferm., Porto Alegre, v. 40, n. e20180347, 2019.

TULLY, M.P., CANTRILL, J.A. The validity of explicit indicators of prescribing appropriateness. Int J Qual Health Care. v.18, n. 2, p.87-94, 2006.

Autor para correspondência:

Adriana Mary Mestriner Felipe de Melo

Email: mestriner@unigran.br

Centro Universitário da Grande Dourados (UNIGRAN)

Recebido: 27/02/2021 Aceite: 19/05/2021 